\title{
Epidural catheter: where does it go?
}

\author{
Esquenazi Najman I. ${ }^{1}$, Duarte Pimentel P. ${ }^{2}$, Almeida Mastache N. ${ }^{2}$, Moreira Petri F. $^{2}$, \\ Magalhães Ramos de Souza F.H. ${ }^{2}$, Pinho Mendes Pereira A.C. ${ }^{3}$ \\ 1-M.D. PhD. Anesthesiologist, Department of Anesthesiology, INCA-HC1, RJ, Brazil \\ 2-M.D. Anesthesia Resident, Department of Anesthesiology, INCA-HC1, RJ, Brazil \\ 3-M.D. Anesthesiologist, Chief of the Residency Program in Anesthesiology, Department of Anesthesiology, INCA-HC1, RJ, Brazil
}

\section{BACKGROUND}

Chemoembolization is a combination of local delivery of chemotherapy and a procedure called embolization to treat cancer. An embolic agent is placed inside the blood vessels that supply blood to the tumor, in effect trapping the chemotherapy in the tumor. Reports on the use of Drug-Eluting Bead Irinotecan (DEBIRI) in transarterial chemoembolization therapy for metastatic colorectal cancer show promising results. In order to reduce the painful response, intravenous sedation associated with epidural analgesia for DEBIRI procedure and postoperative follow-up, can be used. The epidural analgesia for DEBIRI procedure has demonstrated favorable results. Therefore, the success of the epidural analgesic technique is directly related to the proper positioning of the catheter.

\section{CASE REPORT}

A 79-year-old male patient, who has been diagnosed with right colon adenocarcinoma including liver metastasis, was submitted to DEBIRI procedure by an interventional radiologist. After intravenous sedation, epidural catheter was taken place. Epidurography was performed by injecting intratecal contrast through the epidural catheter, just to certify its tip position, prior to morphine and local anesthetic injection. At this moment, it was noticed that the catheter drew a circle appearance, with its tip pointing downward in the direction of the lower limbs. The catheter was then pulled and replaced under direct visualization by fluoroscopy until its tip was pointed up. Successful analgesia was obtained. No complications were observed

\section{DISCUSSION}

Continuous epidural analgesia is considered the gold standard for the management of postoperative pain. Unfortunately, even following the precise technique for introducing the epidural catheter, its blind insertion is not certainly related to its proper positioning. Literature reports that the insertion of excessive amounts of catheter into the epidural space is a risk factor for knot formation. Nevertheless, the optimal amount of catheter to insert into the epidural space, in order to minimize complications, is still a matter of debate. $[1,2]$

\section{LEARNING POINTS}

We aimed to call attention to the occurrence of unexpected positioning of the epidural catheter, possible related with analgesia failures in painful procedures.
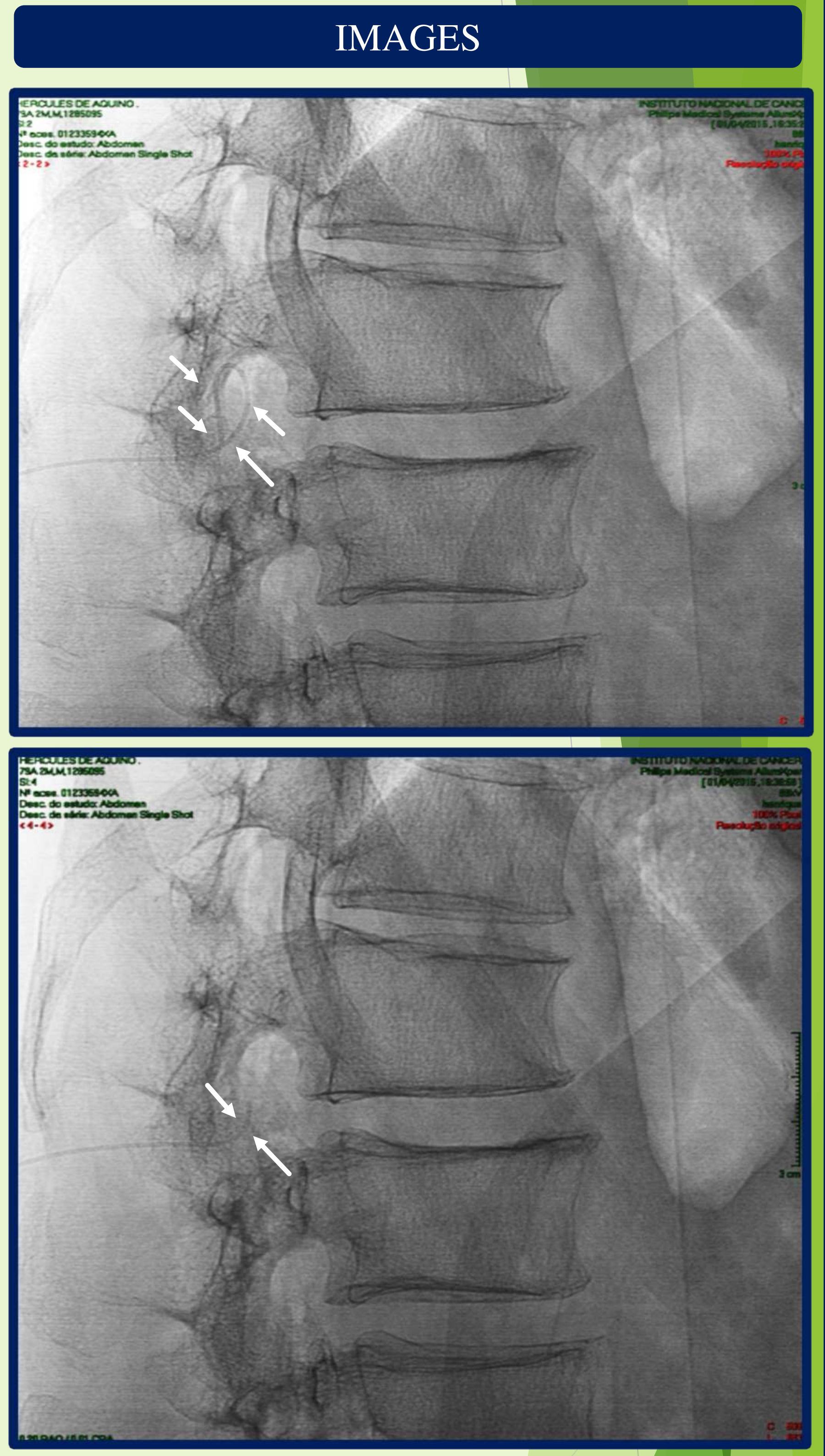

\section{REFERENCES}

[1] Jae-Hang Shim. Strategic Placement of Epidural Catheter. Korean J Anesthesiol. 2012;63(3):193-194.

[2] Renehan EM, Peterson RA, Penning JP, Rosaeg OP, Chow D. Visualization of a looped and knotted epidural catheter with a guidewire. Can J Anaesth. 2000;47:329-33.

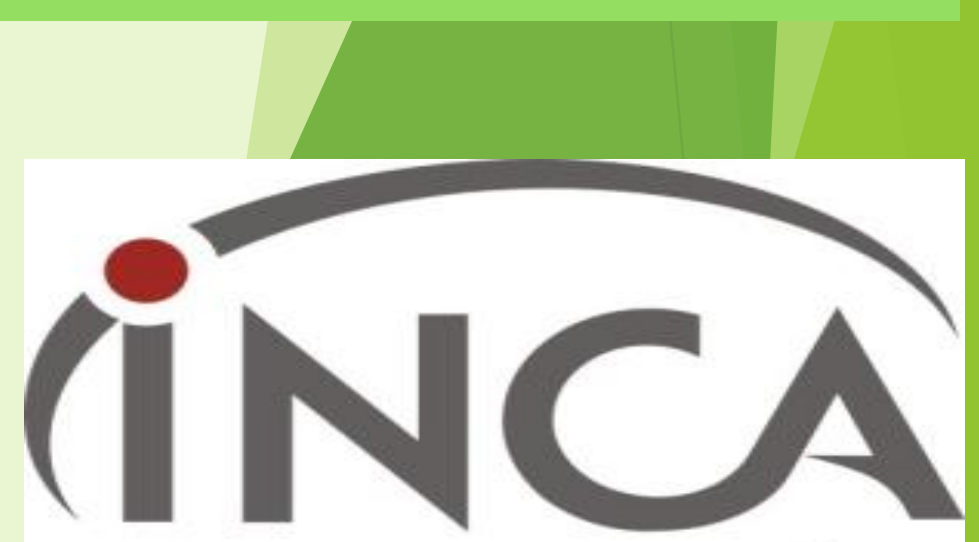

